\section{Metrics for Measuring Disaster Preparedness}

M. Hopmeier; T. Goodwin

Unconventional Concepts, Inc., USA

Considerations in the creation and establishment of a systematic method for evaluation of the actual level of preparedness for medical response will be presented including; (1) the concept of metrics and measures of performance as they apply to response operations involving medical systems; (2) the concepts of "lessons learned" vs. "lessons observed"; (3) standardization and evaluation; and (4) determination of when an organization is "good enough". Additional discussion will focus on: (1) how these factors can be applied to medical disaster response; (2) how metrics can be standardized and evaluated; (3) the role of standards in the United States to involve the Joint Committee on Accreditation of Healthcare Organizations (JCAHO) to assist in development and implementation; (4) determining who are the best users of metrics; and (5) what group of people will benefit the most from the use of metrics.

An enormous amount of time and resources are invested regularly in the development and implementation of exercises to improve the preparedness level of medical systems to deal with a variety of disasters. In the United States (US) in 2003, the passage of the Bioterrorism Preparedness Act (HR 3448) provided states with $\$ 1.5$ billion to achieve the minimum levels of preparedness for a mass-casualty, bioterrorism incident. However, there does not exist an established systematic method to assess and evaluate the actual capabilities or level of preparedness of the medical system. In addition, there lacks a standard set of metrics to effectively assess the response performance during actual incidents (i.e., earthquake response, influenza outbreak).

A "qualitative" review of US mass-disaster exercises from 2000 and 2003, known as the Top Officials Exercise (TOPOFF) was conducted. Surveys of internal and external comments from policy leaders provided key observations. One observation was that there are no universal standards to measure the outcome and success of the exercise.

Keywords: bioterrorism; exercises; medical; metrics; observations; preparedness; standards; system

Prebosp Distust Med 2005:20(2):s72

The Use of an Emergency Response Tabletop Exercise to Foster Collaboration among Regional Public Health Partners and Academia

M. Stephen; ${ }^{1}$ B.D. Devine; ${ }^{2}$ D.D. Dangerfield; ${ }^{2}$

E.G. Gebbie, ${ }^{3}$ W.A. Alvarez,; M.M. McCollum, ${ }^{3}$

J.P. Piotrowski ${ }^{3}$

1. Columbia University Center for Public Health Preparedness, New York, New York, USA

2. New York State Department of Health, New York, New York, USA

3. Columbia University, New York, New York, USA

At the request of the New York State, Department of Health, Metropolitan Area Regional Office (MARO), the Columbia University Center for Public Health Preparedness (CPHP) facilitated an exercise involving the MARO and the county health departments of the Lower Hudson Valley region that was intended to facilitate understanding of each agency's emergency response capabilities and highlight the impact that mutual aid can have on public health preparedness. The event was the culmination of more than two years of relationship building between Columbia University, the MARO and local health departments. During that time, the CPHP developed emergency preparedness training courses and drills with the MARO and the health department partners.

For the latest event, a low-stress, weather emergency scenario was developed by the CPHP for the exercise, with guidance from senior MARO staff. To add an element of realism, the event was held at the State Emergency Management Office's Emergency Operations Center in Poughkeepsie, New York. As the scenario unfolded, a CPHP faculty member asked relevant questions regarding what planning, response, recovery, and evaluation measures each participant and county would take to ensure the safety of their community and staff. The CPHP personnel observed and recorded the reactions of each agency, specifically noting internal and external notification processes and the agencies' roles, responsibilities, and capacities in addressing the needs of local communities. Each participant was given a four-page evaluation form at the conclusion of the exercise and the CPHP staffers completed an eleven-page observation form.

Taken together, the response data provided a clear, informed understanding of the region's emergency preparedness needs and capacities, and highlighted areas for improved cooperation. The importance of collaboration between all levels of public health agencies, their emergency response partners, and academia cannot be underestimated, especially with the new threat of global terrorism.

Recognizing the significance of maintaining and strengthening existing relationships, the CPHP, MARO, and local health departments met to facilitate understanding of regional emergency operations. As a result, many new areas of collaboration were discovered including equipment allocation, regulation enforcement, and communication technology. Participants concluded the exercise better positioned within their agencies and the region to respond not only to acute public health needs, but also to maintain essential public health services during an emergency.

Keywords: emergency; response; tabletop exercises

Prehosp Disast Med 2005;20(2):s7

Systems Approach to Optimizing Disaster Response M. Hopmeier,; ${ }^{1}$ C. Lee, ${ }^{1}$ T. Davis, ${ }^{2}$ J. Lowell, ${ }^{3}$ R. Carmona ${ }^{4}$

1. Unconventional Concepts, Inc., USA

2. Emory University School of Medicine, USA

3. US Department of Homeland Security, USA

4. US Surgeon General, Department of Health and Human Services, USA

Introduction: Medical response to a disaster generally is typified by a rapid introduction of all available materiel and personnel. Unfortunately, it also is typified by a significant amount of wasted assets early during the response period. A need for these wasted assets, then becomes vital during the later stages of a response. 
Immediately after the 11 September 2001 World Trade Center attacks, all available medical personnel rushed to their hospitals with no planning and no reserve. In the event that this had produced mass casualties (versus mass fatality), the medical system would have collapsed completely from exhaustion in 24-36 hours: during the early phase, many of the awake and alerted personnel who responded had very little to do. This disorganization and lack of efficiency possibly could have increased the likelihood of illnesses, injuries, and deaths, due to lack of resources during the later stages of response.

The authors propose assessing a medical response to a disaster from the point of view of optimizing the overall response (while potentially sub-optimizing various subsets or specific aspects) with the end goal of reducing overall mortality and morbidity, not merely during the first 24-48 hours, but throughout the entire response. This assessment will be based on the use of various techniques, including those associated with operations research, as well as those methodologies used for analyzing and optimizing complex processes, such as used by the Toyota Corporation's "Just in Time" manufacturing philosophy.

Methods: Using such a "systems-approach model", a mindset adopted from the engineering and business worlds, the disaster response can be thought of in terms of a system of inputs (personnel, material, skill sets) and outputs (medical services, delivery of milestones) that drive the medical response. Published data of disasters from conventional to biological attacks was charted, and hypothetical scenarios on a time continuum was displayed using a Gannt chart.

Results: Intervals of time denoting the use of specific personnel, skill sets, and materiel are displayed, as well as times rendering medical services and disaster "milestones", or critical points in the response timeline such as arrival of first medical team on scene.

Conclusion: The key benefit derived from this approach is the ability to determine what skill sets and materiel are needed during a response.

Keywords: disaster; mass casualty; medical; preparedness; response; systems approach model

Prebosp Disast Med 2005;20(2):s71-s72

Community-based Therapeutic Care in Ethiopia: A Public Health Approach for the Treatment of Severe Malnutrition

\section{T. Khara; A. Walsh; K. Sadler}

Valid International, United Kingdom

Introduction: Previously, the treatment of severe malnutrition during nutritional emergencies has focused on therapeutic feeding centers. Such centers often are costly, slow to set up, reach only a small proportion of the affected population, and produce high costs for families. The aim of this research is to assess the impact of a new public health approach for the treatment of severe malnutrition.

The principles of community-based, therapeutic care (CTC) are: (1) improved coverage and access to services through decentralizing treatment sites and enabling home- based treatment; (2) timely provision of services and early identification of malnourished children; and (3) improved local capacity to identify and manage acute malnutrition.

Hypothesis: During nutritional emergencies, a CTC strategy can treat severely malnourished children effectively, and quickly achieve high coverage.

Methods: The study was conducted in Northern Ethiopia in 2003-2004. The design was a prospective cohort of all children treated in the Concern Ethiopia CTC program.

The CTC program consists of: (1) mobilization and outreach; (2) the Supplementary Feeding Program (SFP); (3) the Outpatient Therapeutic Program (OTP); and (4) the Stabilization Center (SC).

In mobilization and outreach, traditional structures and mother-to-mother networks mobilized communities and referred children to the program. Employed outreach workers followed-up with children at home.

In the SFP, moderately malnourished children and those discharged from the OTP received a ration of supplementary food and routine medicines fortnightly.

In the OTP, severely malnourished children with appetites and no medical complications were admitted for outpatient treatment. Children attended one of 18 decentralized distribution sites weekly. Sites were set up through local health facilities. Children received a medical and nutritional assessment, ready-to-use therapeutic food (RUTF), standard routine medicines, and treatment for any additional medical conditions.

In the SC, clinical and nutritional criteria (lack of appetite and presence of medical complications) selected children that required inpatient treatment. These children were referred to the district hospital where treatment based on standard World Health Organization protocols for phase one management was provided until appetite returned and complications treated.

Results: From January 2003-January 2004, 590 severely malnourished children ( $<70 \%$ weight-for-height and/or bilateral edema and/or Mid-Upper-Arm Circumference $<11.0 \mathrm{~cm})$ were treated in the CTC program.

Of these, $74.5 \%$ were discharged recovered, $9.7 \%$ defaulted, and $7.5 \%$ died. A further $8.3 \%$ were discharged non-recovered to the SFP after four months in the OTP without attaining discharge weight. In June 2003, coverage was estimated using a stratified design, with strata defined using the centric systematic area sampling approach and active case finding. Coverage was estimated at $77.5 \%$ (95\% Confidence Interval 65.7-86.2).

All these results meet international Sphere standards for therapeutic care.

Conclusion: The CTC performs well against international standards for therapeutic care and can achieve high coverage of the target population with minimal disruption to families.

Keywords: children; families; malnourished; starvation; therapeutic care; weight

Prehosp Disast Med 2005;20(2):s72 